\title{
The Wellness Workshop - A Peer-Led Intervention within Mental Health Services - An Experience Report
}

Terry Bowyer ${ }^{1 *}$, Nick Plumbridge ${ }^{1}$, Neal Beamish ${ }^{1}$, Caroline Wawman $^{2}$ and Ryan Williams ${ }^{2}$

${ }^{1}$ Dorset Mental Health Forum, UK

${ }^{2}$ Dorset Healthcare University Foundation Trust, UK

\begin{abstract}
Summary
The 'Wellness Workshop' is a peer-led intervention within mental health services. It was designed and implemented by two Peer Specialists (people with their own lived experience of mental illness and recovery), with support from two Occupational Therapists, and is aimed at helping people experiencing mental health distress in order to explore, demonstrate and promote the principles of Personal Recovery discussed by Slade (2009). The workshop is a learning model with an emphasis on instructing attendees how to maintain good mental health using a strength-based approach to attaining wellness. The central component and guiding principle is the 'lived experience' of the facilitators who can act as role-models offering their vital experiential knowledge, hope and understanding to the participants. The style of the workshop is conversational and interactive, lasting for approximately one hour. It has received very positive feedback from the participants, who often state that they can better relate to the information and advice delivered by Peer Specialists rather than traditional mental health service staff. The workshop has primarily been delivered at the local NHS Crisis Team Day Hospital, but its popularity with people who access mental health services and traditional nursing staff has resulted in the workshops being delivered at other sites in the county.
\end{abstract}

\section{Origin, Aims and Development}

In 2009 Nick Plumbridge and Terry Bowyer - two people accessing mental health services in Dorset UK - were introduced socially by the Community Psychiatric Nurse that they shared. As they got acquainted and shared their experiences of treatment they realised there was very little peer-led activity within local services. They both felt this was a vital element missing from the treatment pathway. It signalled an opportunity for some new thinking and they came up with the idea for the Wellness Workshop. They discussed the possibility of designing and delivering a session that would function as an intervention for people experiencing mental distress. They wanted to see if they could use their insights into living with a mental health problem to help others.

Their role in this would be one of Peer Specialists-people who are experts by experience and can use this knowledge and learning as front-line workers in the mental health system. The workshop they developed was designed as an addition to traditional services which can complement and enhance what is already offered by existing mental health service staff. This opportunity enables the Peer Specialists to share their unique understanding and perspective of mental distress and to demonstrate directly the principles of Recovery by giving them direct access to people in crisis.

The Peer Specialists who designed the workshop stated their aim: "To inspire hope amongst attendees that wellness is achievable by sharing our knowledge and insight on mental health problems and recovery. To re-assure attendees and help them to understand their predicament and to provide a safe environment to explore important issues relating to their mental health".

The development of the workshop was based on reports discussing the development of inter-active 'learning sets' by people in mental health crisis services [1]. The aims of these 'learning sets' were to pool learning; using existing knowledge and personal experience of mental illness and Recovery in an environment conducive to sharing and reinforcement. This approach underpins the basic premise of the workshop - that reallife mental health experiences can be used therapeutically to aid others.

The content of the workshop was designed around the principles of recovery; health, strength and wellness which are considered to represent good practice in mental health service agendas [2,3]. It was decided the workshop would be conversational and flexible, with the facilitators using their own lived experience of mental illness to demonstrate a shared humanity with the workshop participants in order to promote trust and inspire a more therapeutic engagement than is generally seen within services. It champions the value of psychosocial input and the therapeutic benefit of talking about your mental health with others who understand and can relate to you because they have had similar experiences.

One of the aims of the project was to promote the idea to participants about not just being passive recipients of care but active in their own routes to wellbeing. Recovery is a highly personal and subjective experience - each person's route to getting better is different It is less about being 'fixed' by others than the person learning to selfmanage their condition themself. The core belief of this approach is that the person can be the centre of their own recovery and the answers lie within. The challenge was to help people in crisis by demonstrating this directly using Peer Specialists.

After writing the proposal and getting clearance to proceed, the Peer Specialists initially attended and observed traditional mental health sessions run by Occupational Therapy staff at the local crisis team day hospital to gain an understanding of the challenges faced in delivering material to people in mental health crisis. Use of the expertise of these mental health staff was also helpful in deciding how the proposed workshops could be delivered demonstrating co-production and partnership working, also integral to recovery orientated practice [3].

*Corresponding author: Terry Bowyer, Dorset Mental Health Forum, UK, E-mail: terrybowyer@dorsetmentalhealthforum.org.uk

Received June 27, 2013; Accepted August 09, 2013; Published August 12, 2013

Citation: Bowyer T, Plumbridge N, Beamish N, Wawman C, Williams R (2013) The Wellness Workshop - A Peer-Led Intervention within Mental Health Services - An Experience Report. JBiosafety Health Educ 1: 104. doi:10.4172/2332-0893.1000104

Copyright: $\odot 2013$ Bowyer T, et al. This is an open-access article distributed under the terms of the Creative Commons Attribution License, which permits unrestricted use, distribution, and reproduction in any medium, provided the original author and source are credited. 
The workshop was piloted on four occasions, attended by people in mental health crisis and by staff, including Caroline Wawman and Ryan Williams; two Occupational Therapists who helped support the project and get it off the ground. They provided the necessary clearance, a well-resourced venue, and regular access to people in crisis on a monthly basis. Feedback was gathered from both the attending staff and the workshop participants after each of these early workshops in order to refine the model. Adjustments were made to the content of the workshop accordingly.

\section{Synopsis of the Workshop}

Introduction. Group rules and Confidentiality Disclaimer.

Warm-up exercise: Wellness and Recovery in mental health-what do they mean?

Recovery is a 'journey' - discussion.

Activity- 'Thoughts \& Feelings'- instilling hope for the future.

Acceptance and denial of a mental health condition.

Experts in your own wellness - promoting self-management.

Taking responsibility and level of effort.

Conclusion, summary and final thoughts.

\section{Development of the Workshop}

Since February 2010 the workshop has been delivered on over 50 occasions, with an average of 6 participants in each session. These have mostly been undertaken at the local Crisis Team Day Hospital, but more recently at an older person's day hospital, and within 3 acute settings locally. This means that over 300 people have taken part in the Workshop programme to date. In order to accommodate the expansion in the interest of the workshop additional facilitators have been recruited and trained. All of the trainee facilitators have lived experience' of mental illness, have knowledge and understanding of their own personal recovery, consider themselves well progressed in their own path to wellness, and feel able and to share this experience openly with others.

\section{Feedback}

Although this work is not a research project we recognised the need to capture the perceptions of those involved in the project. Early feedback about the workshops from participants included the use of self-reported quantitative ratings of hopefulness, both before and after the workshop event. Hope is considered as being a crucial early driver in the recovery process and one of the main psycho-dynamics the workshops were designed to target in the people we were delivering to. However, in keeping with the style of the workshop, qualitative narrative statements appear to more accurately capture the experience of the participants. At the end of each workshop we asked the participants to self-report on how they found the session.

The overwhelming response from the participants was that it is highly beneficial to hear about recovery from other people who have actually been through the whole mental health experience. It addresses the gap in empathy so often prevalent between traditional workers and people who use services in our mental health system.

"It was hugely beneficial to have yourself and your colleague talk of your own experiences; first hand knowledge is far more effective than just hearing from medical professionals".
"The presenters understood very well how you feel when you have a mental illness; they made a lot of sense".

"I've learnt it's about taking responsibility for my illness".

The recipients reflected that the background of the facilitators gave the recipients hope about their own recovery:

"It's useful to hear from some-one who's experienced it first hand there is hope for all of us".

"Made me feel like I'm going to make it - they did".

"You taught me how to enjoy life again".

"Please keep doing what you are doing and you will help many people to come".

People also reported on what they had learnt about recovery:

"Useful, friendly, informative, mind-stretching, thought provoking".

"It gave myself and other Service Users a comprehensive insight into recovery and wellbeing...It offered enlightenment and instilled a sense of empowerment".

"It was good to have a discussion about mental health and talk about it, rather than just rely on medication."

Feedback from the attending staff has also been collated, with the overwhelming message echoing the recipients, namely that the message of recovery is especially powerful when it comes from people with lived experience':

"The group worked best when you both related your experience... these things can be said easier by someone who has been through services, rather than staff".

"Overall the group went very well; it was a relaxed informal environment and the service users felt comfortable enough to share their experiences".

"I think the success of the group was summed up particularly well by a group member who stated- "this is the best group I've ever been to".

As a team we have been very pleased with the positive response we have had but we recognise that perhaps our work could benefit from a clinical trial to give us a more scientific evaluation of the impact of these workshops. We may look at designing and implementing this in the future as the program develops.

\section{Lessons Learnt and Future Directions}

Facilitators with 'lived experience' involved in these workshops has added value to the work of mental health service staff, particularly within the Crisis Service. In other mental health community settings where the workshop has been delivered, in which the recipients may have more capacity to engage, sessions have a tendency to take more than one hour as various mental health issues are explored in more detail. In all the sessions we actively encourage the interactive nature of the work and welcome discussion around anything pertinent to the participants - even if it is outside the planned content. This gives the peers a chance to share and relate their experience in a useful way to anything they want to explore.

It is important that two Peer Specialists facilitate the workshop as this allows the opportunity to reflect on the progress of the session and to debrief each other at the end of the session if there were any challenging situations to deal with. The facilitators have also found 
Citation: Bowyer T, Plumbridge N, Beamish N, Wawman C, Williams R (2013) The Wellness Workshop - A Peer-Led Intervention within Mental Health Services - An Experience Report. J Biosafety Health Educ 1: 104. doi:10.4172/2332-0893-0893.1000104

Page 3 of 3

the experience of delivering them to be very rewarding to their own recovery, particularly when individual participants declare after the session how much they benefitted from it. There is a sense that 'giving back' in this way is tremendously therapeutic.

There is considerable scope for future expansion of people with lived experience who can function as Peer Workers in the mental health system to help facilitate the recovery of others who access services. It is an innovative step that many support and would like to see developed in this field. The insight Peer Workers scan share and the engagement they can naturally create is second to none in our opinion. The reality of their recovery stories can be a very powerful and influential motivator for change if delivered correctly.

However, it should be stressed that Peer Workers of this nature should not be seen as to replace the role of traditional mental health service staff, but rather to supplement them. By working in partnership - and recognising the skill set that both have to offer - we can work towards delivering the optimum level of recovery orientated practice and maximise the recovery potential of those we work with.

This is reflected in the final thought the Peer Specialists deliver when we close one of our workshops:
"It is possible to empower yourself to become the driving force in your own recoveries and recapture some of the power over your life. The illness doesn't have to control you - you can control it, and in so doing decide on your own destiny".

We hope that our work will pave the way to new ways of working in the mental health system where the expertise of the service user (as a Peer Specialist) can be utilised in a more empowering and effective way.

\section{Acknowledgements}

We are grateful to Dorset Mental Health Forum (a local independent peer led third sector agency) and Dorset Healthcare University Foundation Trust (DHUFT) for support for the development of the Wellness Workshops.

For full details of the structure and content of the workshop please contact the authors via email.

\section{References}

1. Milne A (2001) Learning Sets - The Crisis Service Development Programme. Mental Health Updates (Mental Health Foundation) 3: 4.

2. Anthony WA (1993) Recovery from mental illness: the guiding vision of the mental health service system in the 1990's. Psychosocial Rehabilitation Journal 16: 11-23.

3. Slade M (2009) Personal Recovery and Mental Illness. A guide for Mental Health Professionals. Cambridge: Cambridge University Press, UK. 